\title{
Estimation of Sensor Input Signals that are Neither Bandlimited Nor Sparse
}

\author{
Lukas Bruderer and Hans-Andrea Loeliger \\ ETH Zurich \\ Dept. of Information Technology and Electrical Engineering \\ 8092 Zürich, Switzerland \\ \{bruderer, loeliger\}@isi.ee.ethz.ch
}

\begin{abstract}
The paper addresses the estimation of the continuous-time input signal to a linear sensor that is given in state-space form. In previous work, Bolliger et al. proposed to model the input signal as (continuous-time) white Gaussian noise and derived a corresponding estimator that is based on Kalman filtering. The present paper elaborates on this new estimator. In particular, it establishes the continuity (or the piecewise continuity) of the estimate, presents a new interpolation formula between samples, complements the Kalman-filter perspective by a Wiener-filter perspective, and demonstrates practicality by numerical experiments.
\end{abstract}

\section{INTRODUCTION}

Let $U(t)$ and $Y(t)$ be the real-valued input signal and output signal, respectively, of some sensor. We are given noisy samples

$$
Y_{k} \triangleq Y\left(t_{k}\right)+Z_{k}
$$

of $Y(t)$ at discrete moments $t_{k}, k \in \mathbb{Z}$, (with $t_{k}<t_{k+1}$ ), where $Z_{k}$ (the measurement noise) are i.i.d. Gaussian random variables that are independent of $U(t)$ and $Y(t)$. From these samples, we wish to estimate $U(t)$.

We will assume that the sensor is given by a finitedimensional stable linear state-space model with state $X \in \mathbb{R}^{n}$ evolving according to

$$
d X(t)=A X(t) d t+b U(t) d t
$$

with $A \in \mathbb{R}^{n \times n}$ and $b \in \mathbb{R}^{n \times 1}$, and with

$$
Y(t)=c^{\top} X(t)
$$

with $c^{\top} \in \mathbb{R}^{1 \times n}$. A number of generalizations of this setting will be indicated at the end of Section IV.

Problems of this sort are usually addressed by beginning with additional assumptions on $U(t)$. A classical approach is to assume that $U(t)$ is low-pass filtered Gaussian noise and to estimate it either by a Wiener filter or by a Kalman smoother [1], [2]. (For the former, we need stationary conditions and regular sampling while the latter requires the low-pass filter to be in state-space form.) More general priors on $U(t)$, such as splines [3], have also been used, but lead to more complex estimators. In another approach, $U(t)$ is assumed to be sparse with respect to some basis, as, e.g., in [4], [5]. Yet another approach is proposed in [6]. The stated problem is also related to equalization problems in communications, cf. [7]-[9].
However, in many practical applications, the actual sensor input signal is, strictly speaking, neither bandlimited nor sparse: better sensors might reveal ever more details in the signal. Nonetheless, we need to cope with the given sensor, as well as we can.

A new approach to such estimation problems was proposed (among other things) by Bolliger et al. in [10], [11]. In this approach, $U(t)$ is modeled as white Gaussian noise (WGN) not because the unknown true input signal is expected to resemble WGN, but to avoid unwarranted assumptions on its spectrum. It is shown in [10], [11] that modeling $U(t)$ as WGN leads to a practical estimator that is easily computed on the basis of forward-backward Kalman filtering/smoothing.

The definition of the estimate $\hat{u}(t)$ from [10], [11] can be paraphrased as follows. For $\Delta>0$, let

$$
\tilde{U}(t, \Delta) \triangleq \frac{1}{\Delta} \int_{t-\Delta}^{t} U(\tau) d \tau .
$$

If $U(t)$ is a continuous signal, then $\lim _{\Delta \rightarrow 0} \tilde{U}(t, \Delta)=U(t)$. Assume now that $U(t)$ is white Gaussian noise. Then, for fixed $t, \tilde{U}(t, \Delta)$ is a well-defined zero-mean Gaussian random variable with variance $\sigma_{U}^{2} / \Delta$ for some constant $\sigma_{U}^{2}>0$. The MAP/MMSE/LMMSE estimate of $\tilde{U}(t, \Delta)$ from observations $\left\{Y_{k}=y_{k}\right\}$ is

$$
\hat{\tilde{u}}(t, \Delta)=\mathrm{E}\left[\tilde{U}(t, \Delta) \mid\left\{Y_{k}=y_{k}\right\}\right],
$$

and $\hat{u}(t)$ is defined as

$$
\hat{u}(t) \triangleq \lim _{\Delta \rightarrow 0} \hat{\tilde{u}}(t, \Delta) .
$$

The existence of this limit was shown in [11]. The practical computation of $\hat{u}(t)$ will be reviewed in Section II.

In this paper, we report several new theoretical and experimental results on this estimator. In the theory part, we show, in particular, that $\hat{u}(t)$ is smooth (i.e., continuous and infinitely often differentiable) between sampling times $t_{k}$. In fact, the smoothness is obvious from a new expression for $\hat{u}(t)$ between sampling times that is attractive also for practical computations. We also show that $\hat{u}(t)$ is continuous even for $t \in\left\{t_{k}\right\}$ if $c^{\top} b=0$. In addition, we give a Wiener-filter version of the estimate (6) that further illustrates the nature of this estimate. 
In the experimental part, we report not only some simulation results, but also measurements with a real sensor in an industrial setting similar to [12].

The proofs of Theorems 2 and 3 use factor graphs as in [11], [13], but factor graphs are not otherwise used in this paper.

The paper is structured as follows. Section II reviews the required background from [11]. The new theoretical results are presented in Sections III and IV, and the experimental results are given in Section VI.

\section{BACKGROUND FROM PREVIOUS WORK}

As shown in [11], the estimate (6) is given by

$$
\hat{u}(t)=\sigma_{U}^{2} b^{\top} \tilde{W}(t)\left(\overleftarrow{m}_{X(t)}-\vec{m}_{X(t)}\right)
$$

with

$$
\tilde{W}(t) \triangleq\left(\vec{V}_{X(t)}+\overleftarrow{V}_{X(t)}\right)^{-1}
$$

The column vectors $\vec{m}_{X(t)}$ and $\overleftarrow{m}_{X(t)}$ in $\mathbb{R}^{n}$ and the matrices $\vec{V}_{X(t)}$ and $\overleftarrow{V}_{X(t)}$ in $\mathbb{R}^{n \times n}$ are defined as follows. For $t_{k}<$ $t \leq t_{k+1}$, the forward message $\vec{\mu}_{X(t)}$ is the function

$$
\vec{\mu}_{X(t)}(x(t))=p\left(x(t) \mid y_{k}, y_{k-1}, \ldots\right),
$$

i.e., the probability density of $X(t)$ conditioned on the past observations $Y_{\ell}=y_{\ell}, \ell \leq k ; \vec{m}_{X(t)}$ is the mean vector of this density and $\vec{V}_{X(t)}$ is its covariance matrix. Likewise, the backward message $\overleftarrow{\mu}_{X(t)}$ (for $t_{k}<t \leq t_{k+1}$ ) is the likelihood function

$$
\overleftarrow{\mu}_{X(t)}(x(t))=p\left(y_{k+1}, y_{k+2}, \ldots \mid x(t)\right)
$$

which may be viewed (up to a scale factor) as a Gaussian density in $x(t)$ with mean vector $\overleftarrow{m}_{X(t)}$ and covariance matrix $\overleftarrow{V}_{X(t)}$. The estimate (7) remains correct even in the limit when some (or all) eigenvalues of $\vec{V}_{X(t)}$ and/or of $\overleftarrow{V}_{X(t)}$ go to zero or to infinity.

The recursive computation of $\vec{m}_{X(t)}$ and $\vec{V}_{X(t)}$ amounts to the state-prediction recursion of Kalman filtering, and $\overleftarrow{m}_{X(t)}$ and $\overleftarrow{V}_{X(t)}$ may be computed by an analogous backward recursion [11], [13]. (On-line computation of (7) is possible with sufficient look-ahead.)

For numerical computations, it may be preferable to use the formular (7) only for $t \in\left\{t_{k}\right\}$, and to use the new interpolation formula (12) for any intermediate moments $t$. Note also that, in a stationary situation with constant intervals $t_{k+1}-t_{k}$, the covariance matrices $\vec{V}_{X\left(t_{k}\right)}$ and $\overleftarrow{V}_{X\left(t_{k}\right)}$ (and thus $\tilde{W}\left(t_{k}\right)$ ) do not depend on $k$ and may be computed offline, as is usual in Kalman filtering.

We also recall the following variational characterization of $\hat{u}(t)$ from [10], [11]:

Theorem 1. Assume that observations $Y_{k}=Y\left(t_{k}\right)+Z_{k}$ are only available for $k=1,2, \ldots, K$. Then the estimated pair $(\hat{u}(t), \hat{x}(t))$ with $\hat{u}(t)$ as in (7) and with the corresponding state estimate $\hat{x}(t)$ minimizes

$$
\frac{1}{\sigma_{U}^{2}} \int_{-\infty}^{\infty} \hat{u}(t)^{2} d t+\frac{1}{\sigma_{Z}^{2}} \sum_{k=1}^{K}\left(y_{k}-c^{\top} \hat{x}\left(t_{k}\right)\right)^{2} .
$$

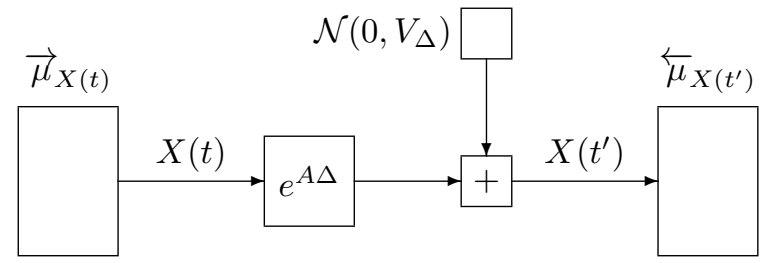

Fig. 1. Factor graph for interpolation with $t^{\prime}=t+\Delta>t$.

among all piecewise continuous ${ }^{1}$ functions $\hat{u}(t)$.

The fact that the estimate (7) is indeed piecewise continuous will be established in Section III.

\section{INTERPOLATION AND CONTINUITY OF $\hat{u}(t)$}

Theorem 2 (Interpolation). Assume that both $t$ and $t^{\prime}=t+\Delta$ lie between adjacent sampling times $t_{k}$ and $t_{k+1}$, i.e., $t_{k}<t \leq t_{k+1}$ and $t_{k}<t^{\prime} \leq t_{k+1}$. Then

$$
\hat{u}\left(t^{\prime}\right)=\sigma_{U}^{2} b^{\top} e^{-A^{\top} \Delta} \tilde{W}(t)\left(\overleftarrow{m}_{X(t)}-\vec{m}_{X(t)}\right)
$$

Note that $\Delta$ may be negative. It is obvious that (12) is a smooth function of $\Delta$, which proves that $\hat{u}(t)$ is smooth between sampling times.

The proofs of the two theorems in this section assume some familiarity with factor graphs [13].

Proof of Theorem 2: From (7), we have

$$
\hat{u}\left(t^{\prime}\right)=\sigma_{U}^{2} b^{\top} \tilde{W}\left(t^{\prime}\right)\left(\overleftarrow{m}_{X\left(t^{\prime}\right)}-\vec{m}_{X\left(t^{\prime}\right)}\right) .
$$

Now consider Fig. 1, which shows a factor graph of the joint probability density of the relevant variables (cf. [11]). Using Tables 2 and 3 of [13] (specifically, equations (II.9), (II.10), (II.12), (III.2), and (III.8) from these tables), we have, first,

$$
\vec{m}_{X\left(t^{\prime}\right)}=e^{A \Delta} \vec{m}_{X(t)},
$$

then $\overleftarrow{m}_{X(t)}=e^{-A \Delta} \overleftarrow{m}_{X\left(t^{\prime}\right)}$ and thus

$$
\overleftarrow{m}_{X\left(t^{\prime}\right)}=e^{A \Delta} \overleftarrow{m}_{X(t)}
$$

and finally $\tilde{W}(t)=e^{A^{\top} \Delta} \tilde{W}\left(t^{\prime}\right) e^{A \Delta}$ and thus

$$
\tilde{W}\left(t^{\prime}\right)=e^{-A^{\top} \Delta} \tilde{W}(t) e^{-A \Delta} .
$$

Inserting these expressions into (13) yields (12).

Theorem 3 (Continuity at sampling times). If $c^{\top} b=0$, then $\hat{u}(t)$ as in (6) is continuous also for $t \in\left\{t_{k}\right\}$.

Conversely, if $c^{\top} b \neq 0$, then $\hat{u}(t)$ is generically not continuous for $t \in\left\{t_{k}\right\}$, as is evident from many examples.

Proof of Theorem 3: We have to show that

$$
\lim _{\Delta \rightarrow 0} \hat{\tilde{u}}\left(t_{k}, \Delta\right)=\lim _{\Delta \rightarrow 0} \hat{\tilde{u}}\left(t_{k}+\Delta, \Delta\right)
$$

\footnotetext{
${ }^{1}$ The qualifier "piecewise continuous" is missing in [11]; in fact, the last step (eq. (33)) of the proof given in [11] is incorrect, but it is correct in [14].
} 
if $c^{\top} b=0$. The relevant part of the factor graph for the lefthand side of (17) is shown in Fig. 2 (top), and the relevant part of the factor graph for the right-hand side of (17) is shown in Fig. 2 (bottom). The former represents the equations

$$
X\left(t_{k}\right)=\tilde{X}\left(t_{k}\right)+b \Delta \tilde{U}\left(t_{k}, \Delta\right)
$$

and

$$
\begin{aligned}
Y\left(t_{k}\right) & =c^{\top} X\left(t_{k}\right) \\
& =c^{\top} \tilde{X}\left(t_{k}\right)+c^{\top} b \Delta \tilde{U}\left(t_{k}, \Delta\right) .
\end{aligned}
$$

If $c^{\top} b=0$, then (20) reduces to $Y\left(t_{k}\right)=c^{\top} \tilde{X}\left(t_{k}\right)$, in which case Fig. 2 (top) is equivalent to Fig. 2 (middle). But for $\Delta \rightarrow 0$, Fig. 2 (bottom) also turns smoothly into Fig. 2 (middle).

\section{Wiener Filter Perspective}

In a stationary situation with $t_{k}=k T$ for fixed $T>0$, the MAP/MMSE/LMMSE estimate (6) can also be obtained via a version of a Wiener filter [1], [2] as follows. Let $G(\omega)$ be the frequency response of the sensor, i.e., the Fourier transform of the impulse response of the system given by (2) and (3). Let $\bar{z}$ denote the complex conjugate of $z \in \mathbb{C}$.

Theorem 4. Under the stated assumptions,

$$
\hat{u}(t)=\sum_{k=-\infty}^{\infty} Y_{k} h(t-k T)
$$

where $h(t)$ is given by its Fourier transform

$$
H(\omega)=\frac{\overline{G(\omega)}}{\sum_{k \in \mathbb{Z}}\left|G\left(\omega+k \frac{2 \pi}{T}\right)\right|^{2}+\frac{\sigma_{Z}^{2}}{\sigma_{U}^{2}}}
$$

Mixed discrete/continuous-time Wiener filters as in (21) are not usually covered in textbooks, and we have not yet been able to find filters similar to (22) in the literature. In any case, deriving a proof from the orthogonality principle of LMMSE estimation [1], [2] is straightforward.

A main point of Theorem 4 is that it further illuminates the nature of the estimate (6). For example, consider the two amplitude responses $|G(\omega)|$ in Fig. 3. The dashed lines in this figure show the aliasing term $\left|G\left(\omega+\frac{2 \pi}{T}\right)\right|$. As long as such aliased parts of $G(\omega)$ remain substantially below the noise-tosignal ratio $\sigma_{Z}^{2} / \sigma_{U}^{2}$, the aliasing does not materially affect the estimate $\hat{u}(t)$.

It should be noted, however, that the Kalman-filter approach (7) is more general than the Wiener filter of Theorem 4. In particular, the Kalman-filter approach works also for nonuniform sampling, and it generalizes easily to time-varying systems (e.g., unstable systems under digital control as in [15]) and to mildly nonlinear systems (via local linearisation as in extended Kalman filtering [1]).
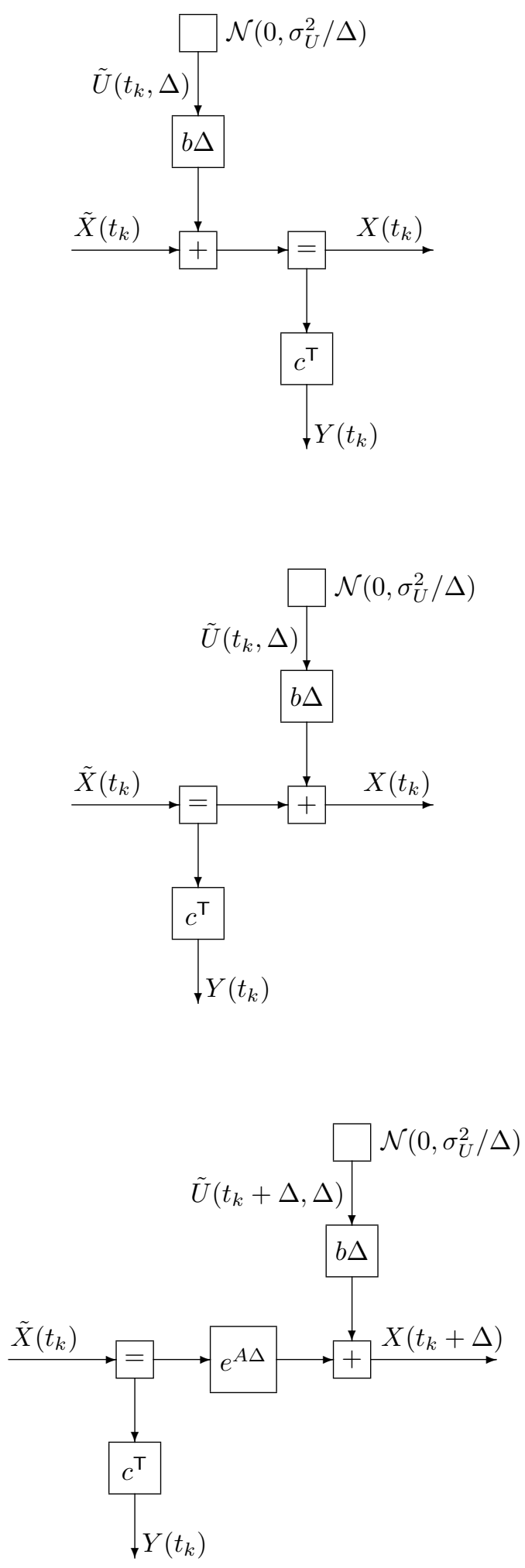

Fig. 2. Factor graph segments used for the proof of Theorem 3. 


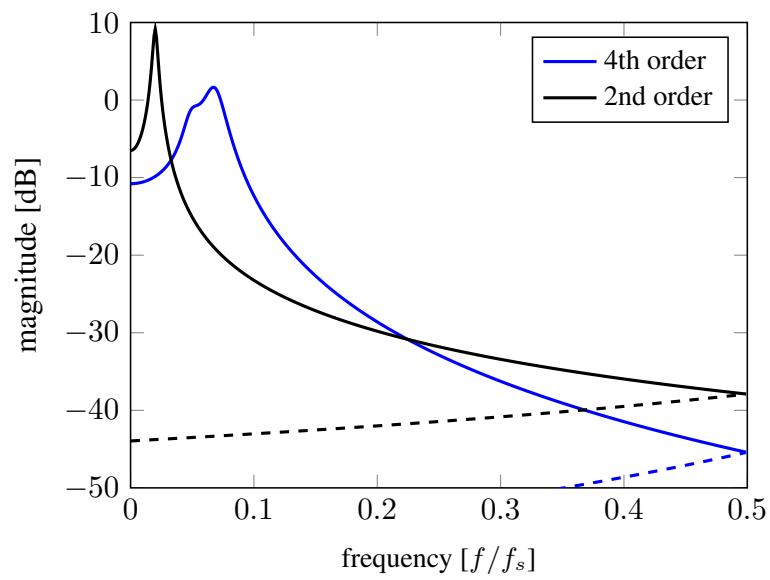

Fig. 3. The amplitude response of two sensors of order 2 and 4 , respectively. The horizontal axis is the normalized frequency $f / f_{\mathrm{s}}$ with $f_{\mathrm{s}} \triangleq 1 / T$. The dashed lines show the aliasing.

\section{Postfiltering}

While the estimate (6) is piecewise continuous (and smooth between sampling times), the user of the sensor may sometimes prefer a smoother-looking estimate of $U(t)$. In this case, the estimate (6) may simply be passed through a suitable lowpass filter (preferably with linear phase response).

Such postfiltering is similar, in effect, to estimating $U(t)$ under the traditional assumption that it is bandlimited. However, the results of the two approaches are not identical, as is easily seen from (22). Moreover, in a Kalman filter setting (as in Sections II and III), the traditional assumption requires a state space model of the noise-shaping filter to be included in the Kalman filter, which increases its complexity; by contrast, postfiltering the estimate (7) does not affect the Kalman filtering at all.

It should be noted that postfiltering (6) is, in principle, a continuous-time operation. A practical time-domain algorithm with piece-wise analytical solutions based on (12) will be discussed elsewhere.

\section{EXPERIMENTAL RESULTS}

Fig. 3 shows the amplitude response of two different sensor models, one of order $n=2$ and the other of order $n=4$, which both originate from fitting low-order models to the measured impulse response of real-world sensors in an industrial setting. The 4 th-order model turns out to satisfy $c^{\top} b=0$ while $c^{\top} b \neq 0$ for the 2 nd-order model. Figs. 4-6 show simulation results with these models for high signal-to-noise ratio $\sigma_{U}^{2} / \sigma_{Z}^{2}$. Note that the input signal in these simulations has effective discontinuities, which is not uncommon for real signals (e.g., forces when moving objects collide). Note also that the input signal is nonnegative, of which the estimator is ignorant.

Fig. 6 shows the input estimate for the 2nd-order model in microscopic detail. It is apparent that the estimated signal is not continuous at the sampling moments, which is due to the fact that $c^{\top} b \neq 0$ in this case (cf. Theorem 3). By contrast, the estimate in Fig. 4 is continuous everywhere in consequence of Theorems 2 and 3.

Fig. 7 illustrates the use of the 4th-order model with measured real-world data. In this case, the true input signal is not known, but a better (more expensive) reference sensor provides a good guess of it. Moreover, the actual sensor dynamics has slightly changed during operation while the estimation uses the unchanged 4th-order model. The estimate is smoothed by postfiltering as in Section V. Due to the uncertainties of the situation, it is difficult to assess the quality of the estimate in absolute terms, but it can certainly be concluded that the estimator works very well in practice.

(The estimated signal in Fig. 7 is sometimes clearly negative. This seems to be due to a bias in the measurement set-up, not due to a problem with the estimator.)

\section{CONCLUSION}

We have presented a number of new theoretical and experimental results on the input-signal estimator that was proposed by Bolliger et al. [10], [11]. In particular, we have established continuity (or piecewise continuity if $c^{\top} b \neq 0$ ) of the estimate, we have complemented the Kalman-filter perspective of [10], [11] with a Wiener-filter perspective, and we have given experimental results that confirm the practicality of the estimator.

\section{REFERENCES}

[1] B. D. O. Anderson and J. B. Moore, Optimal Filtering. Prentice Hall, NJ, 1979.

[2] T. Kailath, A. H. Sayed, and B. Hassibi, Linear Estimation. Prentice Hall, NJ, 2000

[3] G. Wahba, Spline models for observational data. Philadelphia, PA: SIAM, 1990.

[4] P. L. Dragotti, M. Vetterli, and T. Blu, "Sampling moments and reconstructing signals of finite rate of innovation: Shannon meets StrangFix,' IEEE Trans. Signal Proc., vol. 55, no. 5, May 2007, pp. 1741-1757.

[5] M. Mishali, Y.C. Eldar, and A. J. Elron, "Xampling: signal acquisition and processing in union of subspaces," IEEE Trans. Signal Proc., vol. 59, no. 10 , pp. 4719-4734, Oct. 2011.

[6] S. Gillijns and B. de Moor, "Unbiased minimum-variance input and state estimation for linear discrete-time systems," Automatica, vol. 43, pp. 111-116, 2007.

[7] J. G. Proakis, Digital Communications, 5th ed., McGraw-Hill, 2007.

[8] M. Tüchler, A. C. Singer, and R. Koetter, "Minimum mean squared error equalization using a priori information," IEEE Trans. Signal Proc., vol. 50, no. 3, March 2002.

[9] H. Wymeersch, Iterative Receiver Design. Cambridge University Press, 2007.

[10] L. Bolliger, H.-A. Loeliger, and C. Vogel, "Simulation, MMSE estimation, and interpolation of sampled continuous-time signals using factor graphs," 2010 Information Theory \& Applications Workshop, UCSD, La Jolla, CA, USA, Jan. 31 - Feb. 5, 2010.

[11] L. Bolliger, H.-A. Loeliger, and C. Vogel, "LMMSE estimation and interpolation of continuous-time signals from discrete-time samples using factor graphs," arXiv:1301.4793v1.

[12] Y. Altintas and S. S. Park, "Dynamic compensation of spindle-integrated force sensors," CIRP Annals - Manufacturing Technology, vol. 53, no. 1, pp. 305-308, 2004.

[13] H.-A. Loeliger, J. Dauwels, Junli Hu, S. Korl, Li Ping, and F. R. Kschischang, "The factor graph approach to model-based signal processing," Proceedings of the IEEE, vol. 95, no. 6, pp. 1295-1322, June 2007.

[14] L. Bolliger, Digital Estimation of Continuous-Time Signals Using Factor Graphs. PhD Thesis No. 20123 at ETH Zurich, 2012.

[15] H.-A. Loeliger, L. Bolliger, G. Wilckens, and J. Biveroni, "Analog-todigital conversion using unstable filters," 2011 Information Theory \& Applications Workshop, UCSD, La Jolla, CA, USA, Feb. 6-11, 2011. 


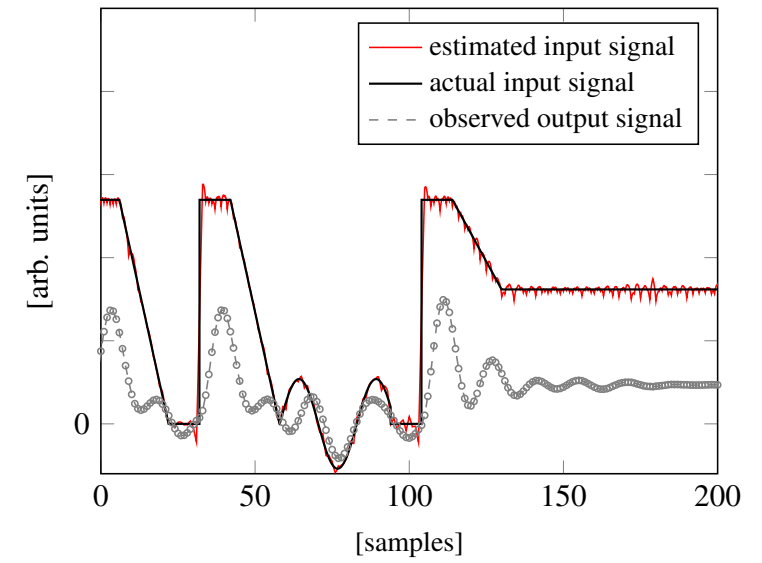

Fig. 4. Input signal estimation (simulation) for 4 th-order model of Fig. 3 with $\sigma_{U}^{2} / \sigma_{Z}^{2}=-41 \mathrm{~dB}$.

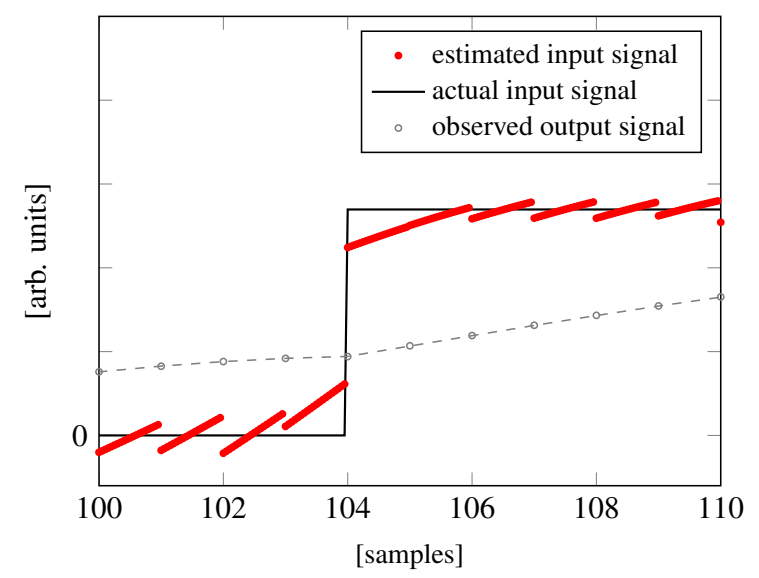

Fig. 6. Close-up of Fig. 5 around a jump of the input signal.

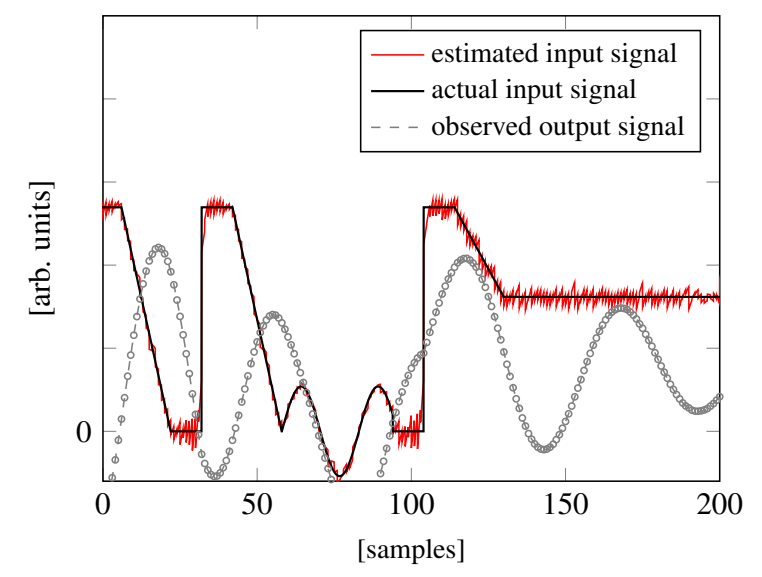

Fig. 5. Input signal estimation (simulation) for 2nd-order model of Fig. 3 with $\sigma_{U}^{2} / \sigma_{Z}^{2}=-31 \mathrm{~dB}$.

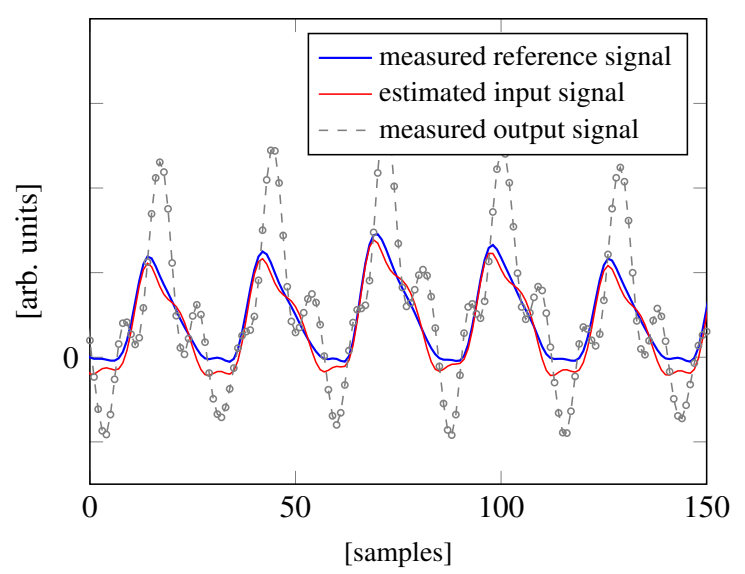

Fig. 7. Input signal estimation from real-world measured data using the 4thorder model of Fig. 3 and postfiltering as in Section V. 